\title{
Constraining input parameters of AMPT model with $\phi$ meson production
}

\author{
Katyayni Tiwari and Md. Nasim \\ Department of Physics, Indian Institute of Science Education and Research, \\ Berhampur-760010, India
}

\begin{abstract}
A Multi-Phase Transport Model (AMPT) has been extensively used to understand the physics behind the experimental observation. Like other models, the outcome of the AMPT model depends on the initial parameters. Therefore, one needs to choose suitable initial parameters before using the model. Lund string fragmentation function has been used to create quark-antiquarks pairs in the AMPT model. The Lund string fragmentation parameters determine the yields and transverse momentum $\left(p_{T}\right)$ spectra of particles produced in nucleus-nucleus collisions. The values of Lund string fragmentation parameters were determined by fitting the charged particle yield and $p_{T}$ spectra measured in the experiment. In this paper, we have shown the yield of strange quarks carrying hadrons, e.g. $\phi$ mesons, are more sensitive to Lund string fragmentation parameters compared to non-strange pions. Using $\phi$ meson spectra measured at RHIC, we have obtained new sets of Lund parameters for $\mathrm{Au}+\mathrm{Au}$ collisions at $\sqrt{s_{\mathrm{NN}}}=11.5,39$, and $200 \mathrm{GeV}$. We have found that using the same set of parameters, we can explain $\phi$-meson yield at $\sqrt{s_{\mathrm{NN}}}=$ 39 and $200 \mathrm{GeV}$, however, we need a different set of parameters for $\sqrt{s_{\mathrm{NN}}}=$ $11.5 \mathrm{GeV}$. This suggests that at low energy, $\sqrt{s_{\mathrm{NN}}}=11.5 \mathrm{GeV}$, the underlying mechanism for particle production is different compared to top RHIC energies. We have also predicted invariant yield of $\pi$ and $\phi$ mesons as a function of $p_{T}$ in $\mathrm{U}+\mathrm{U}$ collisions at $\sqrt{s_{\mathrm{NN}}}=196 \mathrm{GeV}$ to be measured by STAR experiment.
\end{abstract}

Keywords: Heavy-ion Collision, strange quarks 


\section{Introduction}

The Relativistic Heavy Ion Collider (RHIC) has undertaken a Beam Energy Scan program (BES) to look for a change in observations of various measurements as a function of beam energy to establish the partonic phase at higher energy collisions [1, 2]. The first phase of the Beam Energy Scan program (BES-I) plus the top energy at RHIC has allowed access to a region of the QCD phase diagram covering a range of baryon chemical potential $\left(\mu_{B}\right)$ from 20 to $420 \mathrm{MeV}$ [2]. In such a program, the energy dependence of $\phi(s \bar{s})$ meson production plays a crucial role, since it has small hadronic interaction cross-section and freeze-out early compared to other non-strange hadrons [3, 4, 5]. The first phase of the beam energy scan program has been completed and the results are being available to understand the properties of produced matter created in heavy-ion collisions [6, 7, 8, 9, 10, 11, 12, 13, 14, 15, 16]. Various observables are compared to theoretical calculations to understand the physical mechanism behind the measurements [17, 18, 19, 20, 21, 22, 23, 24, 25, 26, 27, 28, 29, 30, 31, 32, 33, 34, 35. One of the frequently used models in heavy-ion collisions is AMPT [36]. The motivation of this paper is to find out suitable input parameters of AMPT model which explains the production of non-strange $(\pi)$ and strange mesons $(\phi)$ measured by the STAR experiment at top RHIC and BES energies and give a prediction for $\pi$ and $\phi$ meson $p_{T}$ spectra in $\mathrm{U}+\mathrm{U}$ collisions at $\sqrt{s_{\mathrm{NN}}}=$ $196 \mathrm{GeV}$.

The paper is organized as follows. In the next section, we discuss the AMPT model. We also discuss the implementation of $\mathrm{U}+\mathrm{U}$ collision in the AMPT model. Section 3 presents the results, which include the comparison of data and model calculation for $\pi$ and $\phi$ meson invariant yield as a function of $p_{T}$. Finally, in section 4 we present a summary of our findings.

\section{The AMPT Model}

The AMPT uses the same initial conditions as in HIJING [37]. However, the minijet partons are made to undergo scattering before they are allowed to fragment into hadrons. In AMPT default model, hadrons are formed from these quarks and antiquarks by using a symmetric fragmentation function $f(z) \propto z^{-1}(1-z)^{a} \exp \left(-b m_{T}^{2} / z\right)$, where $z$ denotes the light-cone momentum fraction of the produced hadrons with respect to that of the fragmentation string and $a$ and $b$ are free parameters [38]. The string-melting (SM) version 
of the AMPT model (labeled here as AMPT-SM) is based on the idea that for energy densities beyond a critical value of $\sim 1 \mathrm{GeV} / \mathrm{fm}^{3}$, it is difficult to visualise the coexistence of strings (or hadrons) and partons. Hence the need to melt the strings to partons. This is done by converting the mesons to a quark and antiquark pair, baryons to three quarks, etc. The quark-antiquark pair production from string fragmentation in the Lund model is based on the Schwinger mechanism [39]. In the Schwinger mechanism, the production probability of the quark-antiquark pair is proportional to $\exp \left(-\pi m_{T}^{2} / k\right)$, where $m_{T}$ is the transverse mass of the quark and $k$ is the string tension, approximately as given by $k \propto 1 /[b(2+a)]$. The AMPT model with string melting leads to hadron formation using a quark coalescence model. The subsequent hadronic matter interaction is described by a hadronic cascade, which is based on a relativistic transport (ART) model [40].

The values of Lund string fragmentation parameters ( $a$ and $b$ ) were determined by fitting the charged particle yield and $p_{T}$ spectra measured in the experiment for different collisions system [36, 41, 42]. In this work, we included the production of strange quarks carrying hadrons, $\phi$ meson, as well as non-strange $\pi$ meson to determine the Lund parameters, $a$ and $b$, for $\mathrm{Au}+\mathrm{Au}$ collisions at $\sqrt{s_{\mathrm{NN}}}=11.5,39$, and $200 \mathrm{GeV}$. We have used two sets for parameters; set 1: $a=0.55$ and $b=0.15$ (proposed in ref. [41]) and set 2 $: a=2.2$ and $b=0.15$. The parton-parton cross-section is taken to be $3 \mathrm{mb}$ (using strong coupling constant $\alpha_{S}=0.33$ ) for both set 1 and set 2 . The termination time of the hadronic cascade is taken to be $30 \mathrm{fm} / \mathrm{c}$.

In the public version of the AMPT model, the deformation parameter of Uranium nucleus is not implemented. In this work, we have implemented deformed shape of the Uranium nucleus in the AMPT model to study the $\mathrm{U}+\mathrm{U}$ collisions. The nucleon density distribution is parametrized as a deformed Woods-Saxon profile.

$$
\begin{gathered}
\rho=\frac{\rho_{0}}{1+\exp ([r-R] / a)}, \\
R=R_{0}\left[1+\beta_{2} Y_{2}^{0}(\theta)+\beta_{4} Y_{4}^{0}(\theta)\right],
\end{gathered}
$$

Where $\rho_{0}$ is the normal nuclear density and $Y_{m}^{l}(\theta)$ denote spherical harmonics and $\theta$ is the polar angle with respect to the symmetry axis of the nucleus. We have used the radius of the nucleus $R_{0}=6.81 \mathrm{fm}$ and the surface diffuseness parameter $a=0.55 \mathrm{fm}$ for $\mathrm{U}$ nucleus. The deformation parameters in the WoodsSaxon profile are $\beta_{2}=0.28$ and $\beta_{4}=0.093$ [43, 44]. 

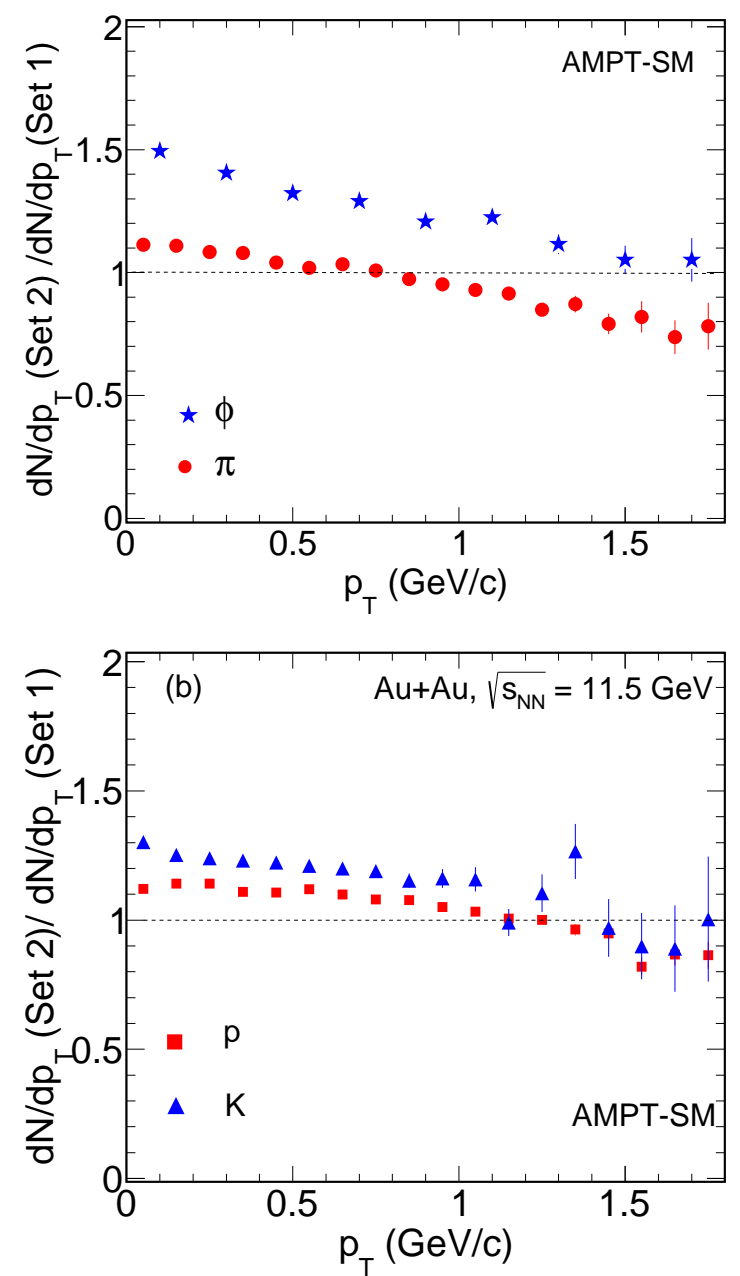

Figure 1: (Color online) (a) Ratios of pions and $\phi$ mesons $p_{T}$ spectra at mid-rapidity between two sets of input parameters of AMPT in Au+Au collisions at $\sqrt{s_{\mathrm{NN}}}=11.5$ $\mathrm{GeV}$. (b) Ratios of kaons and protons $p_{T}$ spectra at mid-rapidity between two sets of input parameters of AMPT in $\mathrm{Au}+\mathrm{Au}$ collisions at $\sqrt{s_{\mathrm{NN}}}=11.5 \mathrm{GeV}$. Here set 1 corresponds to $a=0.55$ and $b=0.15$ and set 2 corresponds to $a=2.2$ and $b=0.15$. 


\section{Results}

\subsection{Au+Au collisions}

We have calculated the transverse momentum spectra of pion and $\phi$ meson using the AMPT model with two sets of parameters, set 1 and set 2. Figure 1 (a) shows the ratios of pions and $\phi$ mesons spectra between two sets of input parameters in AMPT. The solid red circle and solid blue star marker correspond to $\pi^{-}$and $\phi$ mesons, respectively. This calculation is done for $10-40 \% \mathrm{Au}+\mathrm{Au}$ collisions at $\sqrt{s_{\mathrm{NN}}}=11.5 \mathrm{GeV}$. From Fig. 1, we can see that change in $\pi$ spectra is within $15 \%$, however, a large difference ( $\sim 50 \%$ at low $p_{T}$ ) is observed for $\phi$ meson spectra. A similar comparison has been shown for kaons (consist of one strange quark) and protons (similar mass as $\phi$ ) in Fig 1 (b), where a change in spectra is found to be $\sim 20-25 \%$ and 10 $15 \%$, respectively. This study suggests that yield of strange quarks carrying hadrons are more sensitive observable to constraint the Lund fragmentation parameter. These observations are energy independent. In this paper, we have used $p_{T}$ spectra of pions and $\phi$ meson simultaneously to extract Lund fit parameters for $\mathrm{Au}+\mathrm{Au}$ collisions at $\sqrt{s_{\mathrm{NN}}}=11.5,39$ and $200 \mathrm{GeV}$.

Figure 2(a) and (b) compare the experimentally measured $p_{T}$ spectra of non-strange pion and $\phi$ meson with AMPT model calculation in $\mathrm{Au}+\mathrm{Au}$ collisions at $\sqrt{s_{\mathrm{NN}}}=200 \mathrm{GeV}$ [45, 46]. The measurement has been done at mid-rapidity, $|y|<0.1$ (for $\pi$ ) and $|y|<0.5$ (for $\phi$ ). Comparison is shown for three different centralities, 0-5\% (most central), 20-30\% (mid-central) and $60-70 \%$ (peripheral). Lund parameters $(a=0.55$ and $b=0.15)$, used to

fit charged particle spectra in $\mathrm{Au}+\mathrm{Au}$ collisions at $\sqrt{s_{\mathrm{NN}}}=200 \mathrm{GeV}$ [41], are also used here for both pions and $\phi$ meson. We can see AMPT model calculations, with Lund parameters $a=0.55$ and $b=0.15$, explain both nonstrange $\pi^{-}$and strange $\phi$ meson spectra for all $p_{T}$ and all centralities.

After observing good agreement between data and model using parameter $a=0.55$ and $b=0.15$ (set 1 ), we wanted to check what happens if we lower the center-of-mass energy. In Fig. 3, we have shown comparison of $\pi^{-}$and $\phi$ meson spectra between data and AMPT model using parameter $a=0.55$ and $b=0.15$ (set 1 ) at $\sqrt{s_{\mathrm{NN}}}=39 \mathrm{GeV}$. We can see, in most central and mid-central collisions AMPT model explains the data very well for both $\pi^{-}$ and $\phi$ mesons. However, model over predicts the data at high $p_{T}$ for most peripheral collisions.

Figure 4 shows comparison of $\pi^{-}$and $\phi$ meson spectra between data and AMPT model for $\mathrm{Au}+\mathrm{Au}$ collisions at $\sqrt{s_{\mathrm{NN}}}=11.5 \mathrm{GeV}$. Here we can see, 

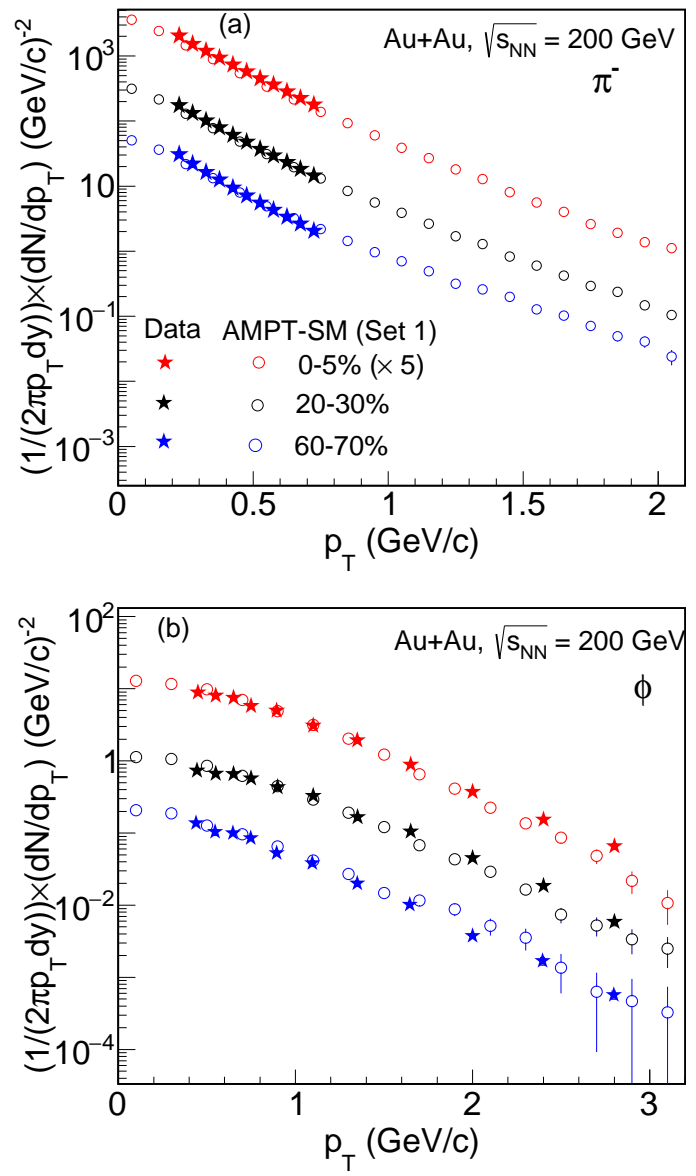

Figure 2: (Color online) Invariant yield of (a) $\pi^{-}$and (b) $\phi$ at mid-rapidity as a function of transverse momentum in $\mathrm{Au}+\mathrm{Au}$ collisions at $\sqrt{s_{\mathrm{NN}}}=200 \mathrm{GeV}$ for $0-5 \%, 20-30 \%$ and 60-70\% centralities. Solid star marker represents experimentally measured values [45, 46] and open circle represents AMPT model calculation using $a=0.55$ and $b=0.15$ (set 1). 
Lund parameter $a=0.55$ and $b=0.15$ (set 1) explains $\pi^{-}$spectra at most and mid-central collisions. However, AMPT model calculation using Lund parameter $a=0.55$ and $b=0.15$ (set 1) under-predicts measured $\phi$ meson spectra at most and mid-central collisions. We have also shown AMPT model results using Lund parameter $a=2.2$ and $b=0.15$ (set 2) in Fig. 4. We do not see any significant change in $\pi^{-}$spectra; however, the comparison between data and AMPT model with set 2 parameter looks better than that of set 1 for $\phi$ meson. This clearly shows $\phi$ meson is a better probe to have control over the Lund parameter. It is important to note that, using the same set of parameters (set 1), one can explain data at both 200 and $39 \mathrm{GeV}$, however. we need a different set of parameters in order to describe data at $11.5 \mathrm{GeV}$. This suggests that the underlying mechanism for particle production at 11.5 $\mathrm{GeV}$ could be different than that of at 39 and $200 \mathrm{GeV}$. This finding is consistent with the finding by the STAR experiment as presented in ref. [11].

\section{2. $U+U$ collisions}

After obtaining suitable sets of parameters of the AMPT model in $\mathrm{Au}+\mathrm{Au}$ collisions at top RHIC and BES-energies, we have calculated the invariant yield of $\pi^{-}$and $\phi$ mesons spectra in $\mathrm{U}+\mathrm{U}$ collisions at $\sqrt{s_{\mathrm{NN}}}=196 \mathrm{GeV}$ for various centralities. The $\mathrm{U}+\mathrm{U}$ collisions are expected to produce more extreme conditions of QCD matter at higher density and/or greater volume than the spherical gold nuclei at the same incident energy. Since Uranium has quadrupole deformed shape, the collisions between Uranium nuclei will produce different initial geometry compared to $\mathrm{Au}+\mathrm{Au}$ collisions. Figure 5 shows the Invariant yield of (a) $\pi^{-}$and (b) $\phi$ at mid-rapidity as a function of transverse momentum in $\mathrm{U}+\mathrm{U}$ collisions at $\sqrt{s_{\mathrm{NN}}}=196 \mathrm{GeV}$ for $0-5 \%$, $20-30 \%$ and $60-70 \%$ centralities. We also compared the results with that of $\mathrm{Au}+\mathrm{Au}$ collisions at $\sqrt{s_{\mathrm{NN}}}=200 \mathrm{GeV}$. In this calculation, we have used Lund parameters $a=0.55$ and $b=0.15$ (set 1) which explain data for $\mathrm{Au}+\mathrm{Au}$ collisions at $\sqrt{s_{\mathrm{NN}}}=200 \mathrm{GeV}$. It is found that the invariant yield of $\pi^{-}$and $\phi$ is higher $(20 \%)$ in $\mathrm{U}+\mathrm{U}$ collisions compared to that in $\mathrm{Au}+\mathrm{Au}$ collisions. This could be due to the higher initial energy density in $U+U$ collisions compared to $\mathrm{Au}+\mathrm{Au}$ collisions. These AMPT results can be compared to the experimental results to be available soon from STAR collaboration. 

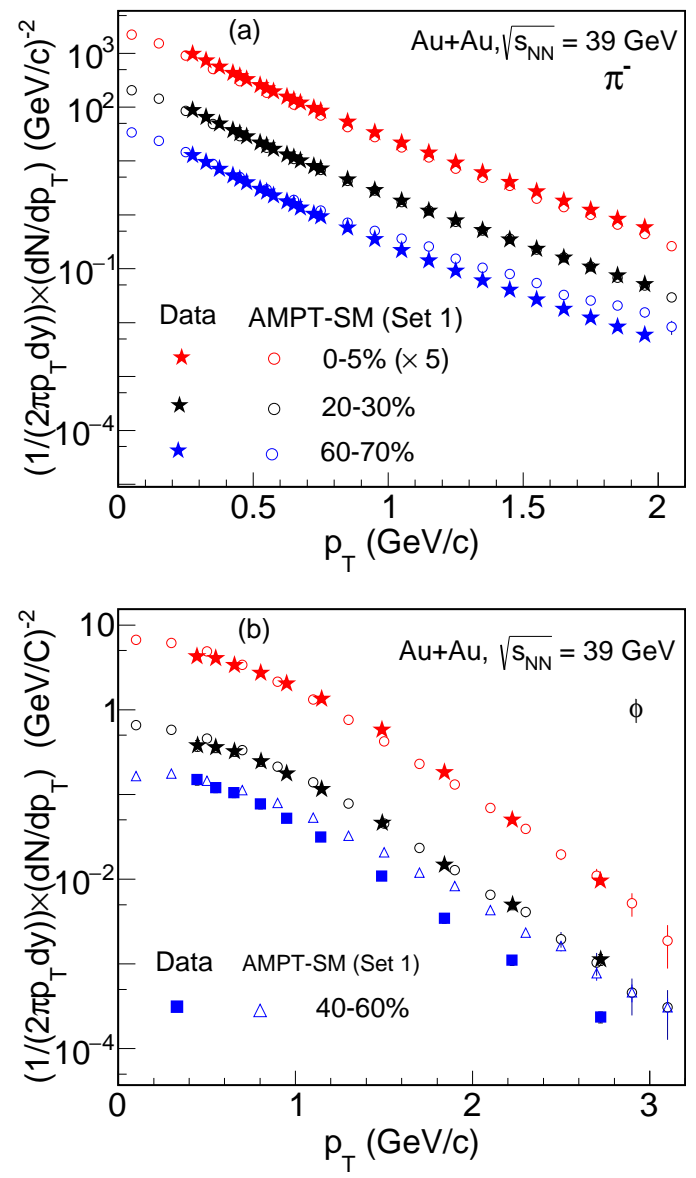

Figure 3: (Color online) Invariant yield of (a) $\pi^{-}$. and (b) $\phi$ at mid-rapidity as a function of transverse momentum in $\mathrm{Au}+\mathrm{Au}$ collisions at $\sqrt{s_{\mathrm{NN}}}=39 \mathrm{GeV}$ for $0-5 \%, 20-30 \%$ and $60-70 \%$ (40-60\% for $\phi$ ) centralities. Solid star marker represents experimentally measured values [12, 11] and open circle represents AMPT model calculation using $a=0.55$ and $b$ $=0.15($ set 1$)$ 

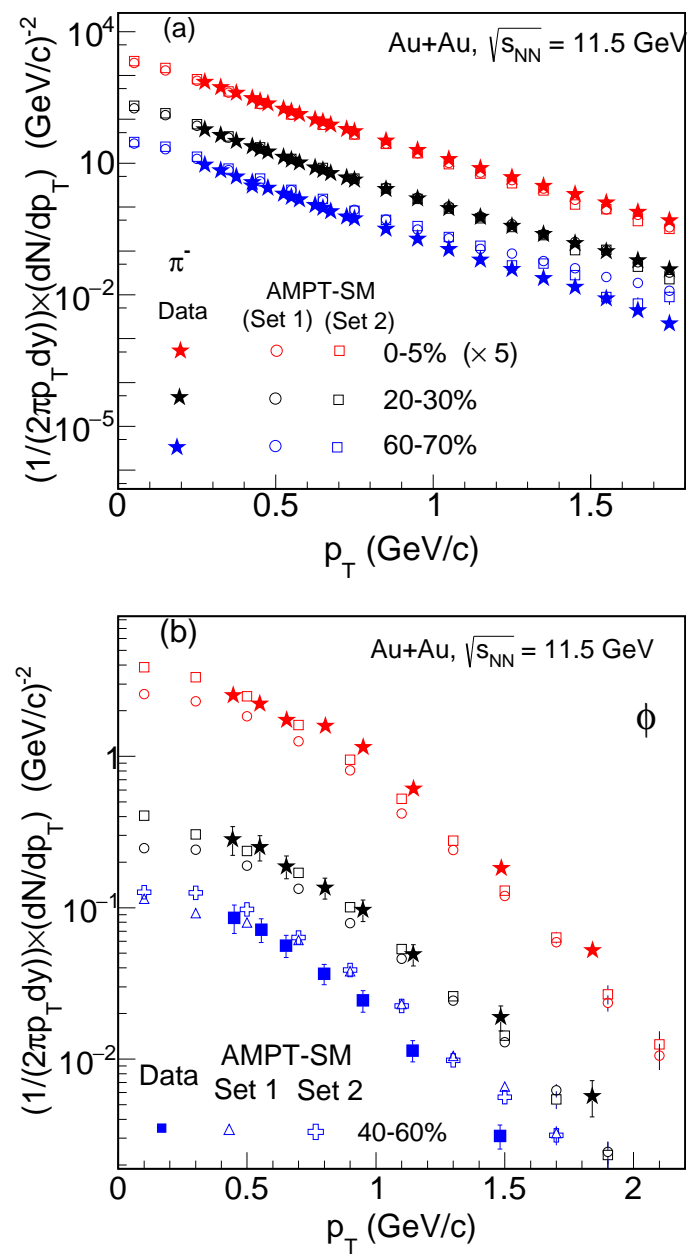

Figure 4: (Color online) Invariant yield of (a) $\pi^{-}$. and (b) $\phi$ at mid-rapidity as a function of transverse momentum in $\mathrm{Au}+\mathrm{Au}$ collisions at $\sqrt{s_{\mathrm{NN}}}=11.5 \mathrm{GeV}$ for $0-5 \%, 20-30 \%$ and $60-70 \%$ (40-60\% for $\phi)$ centralities. Solid star marker represents experimentally measured values [12, 11] and open circle represents AMPT model calculation using parameters set $1(a=0.55$ and $b=0.15)$ and set $2(a=2.2$ and $b=0.15)$ 

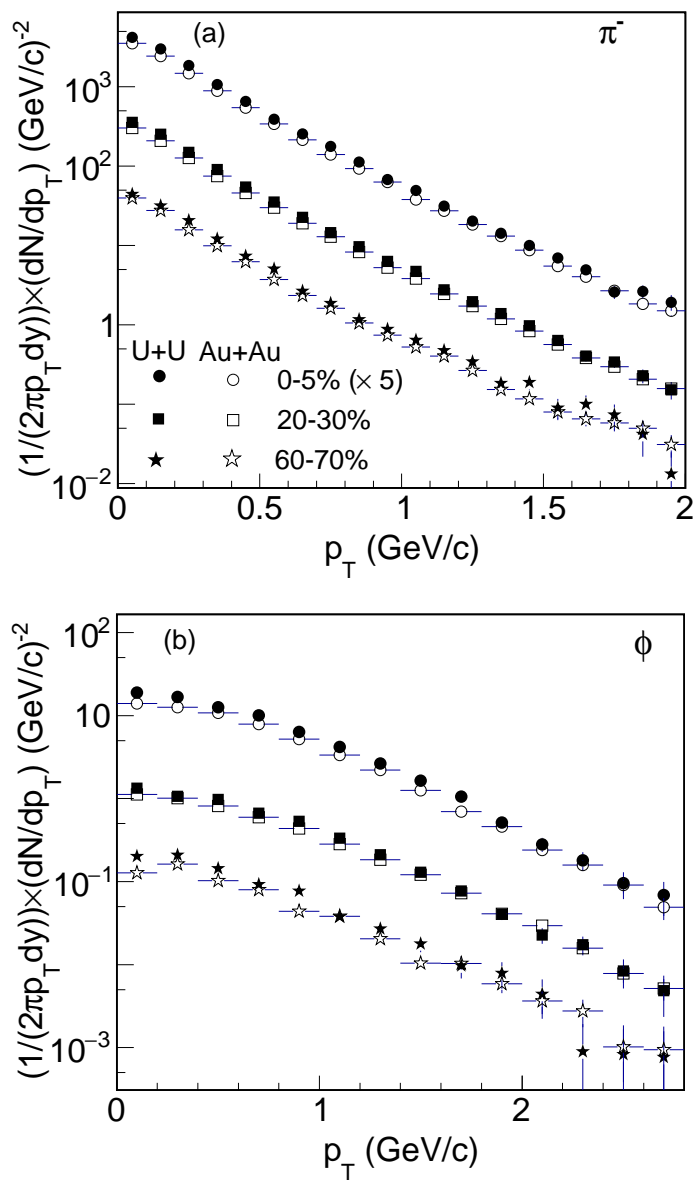

Figure 5: (Color online) Invariant yield of (a) $\pi^{-}$and (b) $\phi$ at mid-rapidity as a function of transverse momentum in $\mathrm{U}+\mathrm{U}$ collisions at $\sqrt{s_{\mathrm{NN}}}=196 \mathrm{GeV}$ for $0-5 \%, 20-30 \%$ and $60-70 \%$ centralities. The AMPT model calculation using parameters set 1 ( $a=0.55$ and $b$ $=0.15$ ) which explains data for $\mathrm{Au}+\mathrm{Au}$ collisions at $\sqrt{s_{\mathrm{NN}}}=200 \mathrm{GeV}$. The AMPT model calculation for $\mathrm{Au}+\mathrm{Au}$ collisions at $\sqrt{s_{\mathrm{NN}}}=200 \mathrm{GeV}$ using same set of parameters are also shown for comparison. 


\section{Summary}

In summary, a compilation of the available data at RHIC energies for the invariant yield of $\pi^{-}$and $\phi$ mesons has been presented. We have shown that strange quarks carrying hadrons are the most sensitive probe to the initial parton dynamics and one should use measured $\phi$ meson yield along with nonstrange particle to determine the model parameters in the AMPT model. It has been found that using the same set of parameters; one can explain both $\pi^{-}$and $\phi$ meson spectra at 200 and $39 \mathrm{GeV}$. However, one needs a different set of input parameters to explain measured $\phi$ meson spectra at $11.5 \mathrm{GeV}$. These results suggest that there is likely a change in the underlying strange quark dynamics in the produced matter during $\mathrm{Au}+\mathrm{Au}$ collisions. In addition, we

have studied the production of $\pi$ and $\phi$ meson in $U+U$ collisions at $\sqrt{s_{\mathrm{NN}}}$ $=196 \mathrm{GeV}$ after implementing deformation for the uranium nucleus in the AMPT model. These results can be compared to experimental measurements at RHIC to understand the particle production mechanism in $\mathrm{U}+\mathrm{U}$ collisions.

\section{References}

[1] STAR Collaboration, B. I. Abelev et al., Phys. Rev. C 81, 024911 (2010).

[2] STAR Collaboration, https://drupal.star.bnl.gov/STAR/starnotes/public/sn0598.

[3] A. Shor, Phys. Rev. Lett. 54, 1122 (1985).

[4] STAR Collaboration, J. Adams et al., Nucl. Phys. A 757, 102 (2005).

[5] B. Mohanty et al., J. Phys. G 36, 064022 (2009).

[6] STAR Collaboration, L. Adamczyk et al., Phys. Rev. C 86, 054908 (2012).

[7] STAR Collaboration, L. Adamczyk et al., Phys. Rev. C 88, 014902 (2013).

[8] STAR Collaboration, L. Adamczyk et al., Phys. Rev. Lett. 110, 142301 (2013).

[9] STAR Collaboration, L. Adamczyk et al., Phys. Rev. Lett. 112, 162301 (2014). 
[10] STAR Collaboration, L. Adamczyk et al., Phys. Rev. Lett. 113, 052302 (2014).

[11] STAR Collaboration, L. Adamczyk et al., Phys. Rev. C 93, 021903 (2016).

[12] STAR Collaboration, L. Adamczyk et al., Phys. Rev. C 96, 044904 (2017).

[13] PHENIX Collaboration, C. Aidala et al., Phys. Rev. C 96, 064905 (2017).

[14] PHENIX Collaboration, C. Aidala et al., Phys. Rev. C 93, 024901 (2016).

[15] PHENIX Collaboration, C. Aidala et al., Phys. Rev. C 93, 011901(R) (2016).

[16] PHENIX Collaboration, C. Aidala et al., Phys. Rev. Lett. 109, 152301 (2012).

[17] Md. Rihan Haque et al., J. Phys. G: 46, 085104 (2019)

[18] Md. Nasim et al., Phys. Rev. C 82, 054908 (2010)

[19] Md. Nasim Phys. Rev. C 89, 034909 (2014).

[20] Md. Nasim et al., Phys. Rev. C 87, 014903 (2013)

[21] C. Zhang et al., Phys. Rev. C 99, 064906 (2019)

[22] Kai Xiao et al., J. Phys. G: Nucl. Part. Phys. 39 (2012) 025011.

[23] K Nayak et al., arXiv:1904.03863.

[24] S. Kundu et al., arXiv:1808.09235.

[25] S. Samanta et al., Phys. Rev. C 97, 015201 (2018).

[26] Z. Yang et al., Phys. Rev. C 95, 014914 (2017).

[27] V. Bairathi et al., Phys. Lett. B754 (2016) 144-150.

[28] S. Cho et al., arXiv:1905.09774 . 
[29] W. Zhao et al., Phys. Rev. C 98, 054905 (2018)

[30] Y. Sun et al., Phys. Lett. B 769 (2017) 219-222

[31] J. Xu et al., Phys. Rev. C 94, 054909 (2016).

[32] H. Li et al., Phys. Rev. C 96, 014901 (2017).

[33] L.-Y. Zhang et al., Phys. Rev. C 99, 054904 (2019).

[34] H. Li et al., Phys. Rev. C 99, 044911 (2019).

[35] S. Lan et al., Phys. Lett. B 780 (2018) 319.

[36] Zi-Wei Lin, C. M. Ko, Phys. Rev. C 65, 034904 (2002); Zi-Wei Lin et al., Phys. Rev. C 72, 064901 (2005).

[37] X. N. Wang and M. Gyulassy, Phys. Rev. D 44, 3501 (1991).

[38] B. Andersson, G. Gustafson, G. Ingelman and T. Sjostrand, Phys. Rep. 97,31 (1983).

[39] J. S. Schwinger, Phys. Rev. 128, 2425 (1962).

[40] B.-A. Li et al., Phys. Rev. C 52, 2037 (1995).

[41] Z. W Lin arXiv:1403.6321

[42] J. Xu and C. M. Ko, Phys. Rev. C 83, 034904 (2011)

[43] Md. R. Haque et al., J. Phys. G: Nucl. Part. Phys. 46, 085104 (2019).

[44] Md. R. Haque et al., Phys. Rev. C 85, 034905 (2012).

[45] STAR Collaboration, B.I. Abelev et al., Phys. Rev. C 79, 034909 (2009 ) ;

[46] STAR Collaboration, B.I. Abelev et al., Phys. Rev. C 79, 64903 (2009). 